\title{
Study on the Comprehensive Testing and Evaluation Technology of Ecological Garden Greenhouse State
}

\author{
Xiangcai Zhu, Yuncai Luan \\ School of Information Science and Technology, Taishan University (TSU), Tai'An 271021, China \\ zhuxiangc@126.com, yos7689@163.com
}

Keywords: Ecological Garden Greenhouse State, Comprehensive Testing, Evaluation Technology.

\begin{abstract}
In this paper, we study the ecological status of comprehensive testing and evaluation technology of horticultural greenhouse, realize the horticultural greenhouse ecological status of anomaly detection, by using the methods of Dixon and Grubbs greenhouse state data processing; The state matching, radial basis function (RBF) neural network and least-square method on greenhouse state data further processing; Using fault tree analysis method (FTA) to realize ecological garden greenhouse state comprehensive evaluation. Research results will provide the horticultural greenhouse ecological status of comprehensive testing and evaluation to provide theoretical basis and technological application
\end{abstract}

\section{Introduction}

The detection technology of ecological greenhouse gardening state has become an important research content of computer application, how to ecological gardening greenhouse state parameter detection and make ecological greenhouse gardening steady state security, in order to make the ecological gardening environment biological health growth, this is the ecological management and technical researchers with the technical problems. State detection technology in this area is one of the important and effective means. The domestic and foreign research on this aspect in the detection of single technology has made some achievements, but there are a lot of technology needs to be improved, especially the integrated detection technology needs to be further studied.

Single detection of integrated detection technology can expand the range of state parameters, and increase the accuracy of state information in order to better capture the ecological gardening greenhouse. Although the single detection technology to mature, but because of the ecological greenhouse gardening state has complex diversity, therefore, this study focuses on the detection of integrated greenhouse condition, especially the analysis of the abnormal state of the data, through the application and implementation and improvement of comprehensive detection technology, the final completion of evaluation of the status of intelligent greenhouse. Comprehensive detection technology can realize all kinds of analysis is more accurate than the single detection technology and more reliable data, in order to realize the ecological gardening greenhouse state more scientific detection judgment and decision making.

\section{Significance, Status, Level and Trend Research}

Dynamic detection and evaluation technology of the ecological park is an effective means to implement dynamic detection of greenhouse condition. State information comprehensive detection technology in this paper can expand the scope of the study, the state parameters of the ecological park to increase accuracy, more fully capture the greenhouse environment. In the comprehensive test, preliminary detection of greenhouse environment including temperature, pressure state, humidity, and other parameters of the image; depth detection operation including image calibration, information synchronization and fusion, behavior description etc.. Because of complexity and diversity of greenhouse condition, this paper focuses on how to synthesize all kinds of detection technology to achieve detection accuracy and universality, realize intelligent evaluation of greenhouse condition. 
The subject of ecological greenhouse gardening state comprehensive utilization detection, image calibration, information synchronization and integration, behavior description, key techniques and methods involved include RBF Technology (radial basis function neural network), LSM method (least squares method, FTA (tree analysis method). To achieve comprehensive inspection and evaluation of greenhouse condition using these technologies.

\section{The research status at home and abroad, the level and development trend}

State Technical Analysis. Study on detection and analysis the status of the project has been carried out in foreign countries, Grab and Dixon and other standards achieved good results in the detection of abnormal data in the state. In China, the research on the detection and analysis of state of the State Key Laboratory of China Institute of science and technology has outstanding achievements.

State detection. State detection is based on the behavior analysis of information, makes the image dynamic state detection becomes difficult. Some research institutes are based on integrated detection model, feature based, region based, active contour based on various direction to research, and achieved certain results. A lot of research on the domestic and foreign research institutions in the state of the scene detection, motion detection, and achieved certain results, but the study, less integrated detection and intelligent evaluation on ecological environment of greenhouse horticulture multi technology status.

\section{The research contents and key problems}

Research content. It researches on acquiring state information of ecological environment of green house horticulture. State acquisition can not only obtain the status information, but also can realize the optimization of a large amount of information, so as to improve the real-time state data processing. This paper uses the method of Bayesian modeling and detection of greenhouse ecological, and using MatLab, Grubbs and Dixon, the technology for data processing and analysis of the greenhouse condition. It researches on matching technology target. The goal, essence is to match the over all object, feature selection has a great impact on the choice of matching algorithm, the matching effect and calculation complexity. This paper uses the object as the feature similarity and spindle, using relevant knowledge of the geometric imaging to detect the calculation standards, and further research on the target object by using the RBF method and LSM method.

Technical indicators. This paper studies the greenhouse state (temperature, humidity, image, video etc.) including key technology, comprehensive detection and intelligent evaluation: the abnormal state detection, data processing, by Grab and Dixon; transmission way of state high-definition image matching, in-depth analysis and processing of data by using RBF and LSM methods; implementation intelligent evaluation by FTA method, and provide a reliable basis for the detection of biological growth and greenhouse. The research results of this paper will provide an important basis for comprehensive evaluation and application of intelligent detection and ecological greenhouse.

Characteristic and innovation. The scientific comprehensive detection technology is proposed in this paper is reasonable, the key problems of greenhouse state acquisition, target matching and intelligent evaluation, analysis, identification and evaluation of the abnormal state of a variety of advanced technical methods and comprehensive ecological gardening greenhouse. 1) acquisition of environmental parameters of ecological gardening greenhouse, by Bias, Dixon, Grubbs modeling and data processing status. 2) according to the object plane relation, object matching method. This method is simple, and is not sensitive to occlusion, combined with the histogram feature fusion, to achieve accurate matching, using RBF and LSM method for further processing of data. 3) model, using predictive function model and a state with FTA technology of intelligent evaluation. 4) using Visual Studio development environment (VB.NET, C++), FTA technology to achieve the dynamic evaluation of ecological management and intelligent greenhouse gardening status etc. 


\section{Study on the route}

This paper comprehensive intelligent detection and research of ecological gardening greenhouse state evaluation technology, and realize the dynamic detection of greenhouse environment status. The following detailed research plan: 1) acquisition of the state of the environment ecological greenhouse gardening: this project adopts the method of Bias model to detect moving objects. According to the position detector calibration, detector to determine the distance of the target, realize the positioning of the target image. 2) technology research, comprehensive objective: comprehensive target region and feature point matching method based on complex process, and easily affected by the noise. This project intends to adopt the main target matching method, the spindle can be obtained by the principle of minimum distance and calculation. 3) research on Intelligent Evaluation Technology: to be through the establishment of model and combined with FTA technology to achieve intelligent evaluation. Technical route is shown in figure 1. Implementation of this scheme is shown in figure 2.

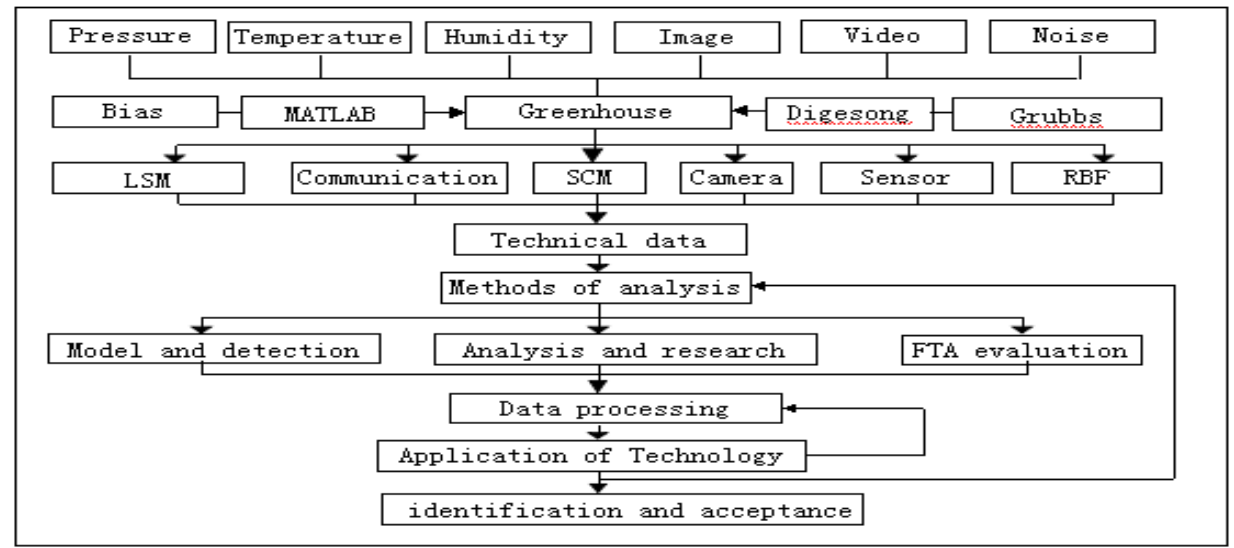

Fig. 1 Technical Route

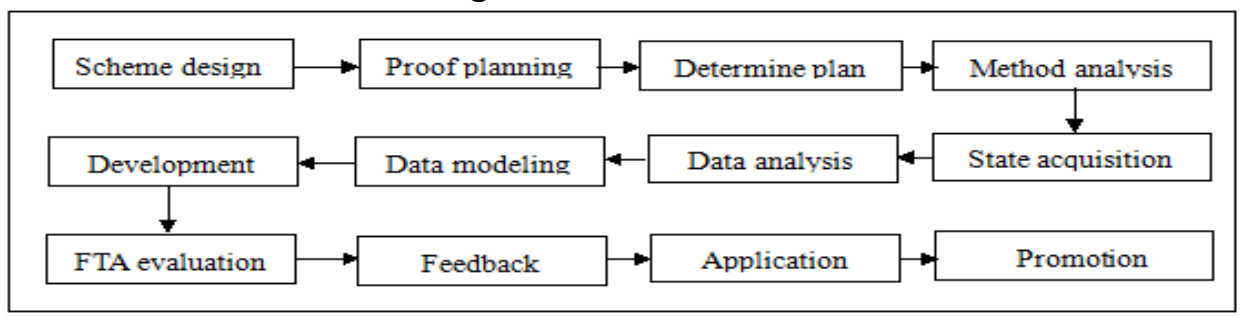

Fig. 2 Implementation plan

\section{Industrialization, support technology}

The development of detection technology makes many research data analysis as the key, comprehensive analysis technology has deep theoretical foundation. Many areas are in need of state detection, that the user can timely understand the operation and troubleshooting. So this project needs a wider market. These research results have good reference value to the research of this paper. This paper intends to study in the following aspects: 1) industrialization to achieve accurate state acquisition. In the accurate detection of the target on the basis of the state, acquisition, classification, and the state is controlled by the position detecting instrument. By Grab J, Dixon criterion of cyclic search data and automatically eliminate the abnormal state. 2) to achieve the goal of matching. To break the regional and deficiency of traditional matching method based on the target, the target matching method based on the spindle, combined with color histogram, the features are fused to achieve accurate matching. And the use of RBF and LSM method for incomplete data repair. 3) intelligent evaluation. Intelligent evaluation of detection in the sequence, the evaluation grade. By using the FTA method of abnormal data on the ecological greenhouse gardening state of the qualitative and quantitative analysis, revealing the reason of abnormal state and abnormal scientific prevention of ecological greenhouse, makes the biological health growth. 


\section{Conclusions}

This paper proposed the detection of integrated ecological gardening reasonable, mainly to solve the key problems of ecological gardening greenhouse state acquisition, target matching and intelligent evaluation, and carries on the analysis, identification and evaluation of abnormal behavior of the ecological state of horticultural greenhouses using a variety of advanced technology. To solve the following problems: 1) the ecological greenhouse gardening technology by the state acquisition, Bias, Dixon, Grubbs modeling and processing. 2) according to the corresponding relationship between the greenhouse ecological gardening plane, the ecological state of the target, greenhouse gardening. This method is simple, combining with the histogram feature fusion, achieve accurate matching. 3) the model was established and used to realize the intelligent assessment of the ecological status of horticultural greenhouses combined with FTA model prediction function and other functions. Study on comprehensive detection technology, to solve the key problems of ecological greenhouse horticulture status, object matching and intelligent evaluation etc. Based on histogram features, feature fusion to achieve accurate matching; using FTA technology to realize the intelligent assessment of the ecological status of greenhouse horticulture, etc.

\section{Acknowledgements}

This research has been partially supported by the national Spark Program project (No.: 2014GA740055); science and technology development project of Tai'an city (No.: 20140630-6); science and technology plan project of Shandong province (No.: J14LN68); the natural science foundation of Shandong province (No.: ZR2013FQ029).

\section{References}

[1] M.H. Tan and Ranganath S.: Communications and Signal Processing, vol.3, 2003, p.1335-1340. (In Chinese)

[2] X.C. Zhu, M.X. Liu and L. Zhang: International Conference of China Communication (Nanning City, the Guangxi Zhuang Autonomous Region,China 2010): P.152-155. (In Chinese)

[3] X.C. Zhu, Y.K. Hou, J. Xu and Y.C. Luan: Instrumentation, Measurement, Circuits and Systems Advances in Intelligent and Soft Computing(Hong Kong, China 2011), vol.127, 201 1, P.867-874. (In Chinese)

[4] Florence B, Sophie M and Jean-Claude P: Algorithm and experiments. Transportation Research, Part C, Emerging Technologies, 2006, 14C (1), P.18-38.

[5] Mihaela P, Alexandru B and Mircea D. : WSEAS Transactions on Advances in Engineering Education, 2006, 3 (5), P.304-311.

[6] X.Y. Lin, A.D. Liu and Y . Li : Applied Mathematics and Computation, 2006, 172 (1), P.188-197. (In Chinese) 